\title{
Periodic Solutions for Impulsive Stochastic BAM Neural Networks with Time-Varying Delays in Leakage Terms
}

\author{
Li Yang and Yongkun Li \\ Department of Mathematics, Yunnan University, Kunming, Yunnan 650091, China \\ Correspondence should be addressed to Yongkun Li; yklie@ynu.edu.cn
}

Received 27 April 2013; Accepted 18 July 2013

Academic Editor: Yong Zhou

Copyright (C) 2013 L. Yang and Y. Li. This is an open access article distributed under the Creative Commons Attribution License, which permits unrestricted use, distribution, and reproduction in any medium, provided the original work is properly cited.

By using an integral inequality, we establish some sufficient conditions for the existence and $p$-exponential stability of periodic solutions for a class of impulsive stochastic BAM neural networks with time-varying delays in leakage terms. Moreover, we present an example to illustrate the feasibility of our results.

\section{Introduction}

Since it was proposed by Kosko (see [1]), the bidirectional associative memory (BAM) neural networks have attracted considerable attentions due to their extensive applications in classification of patterns, associative memories, image processing, and other areas. In the past few years, many scholars have obtained lots of good results on the dynamical behaviors analysis of BAM neural networks. The reader may see [2-8] and the references therein.

But in a real nervous system, it is usually unavoidably affected by external perturbations which are in many cases of great uncertainty and hence may be treated as random. As pointed out by Haykin [9], in real nervous systems, synaptic transmission is a noisy process brought on by random fluctuations from the release of neurotransmitters and other probabilistic causes. And the stability of neural networks could be stabilized or destabilized by some stochastic inputs [10]. Therefore, it is significant and of prime importance to consider the dynamics of stochastic neural networks. With respect to stochastic neural networks, there are many works on the stability. For example, in [11-17], the scholars studied the stability of different classes of stochastic neural networks. For other results on stochastic neural networks, the reader may see [18-23] and the references therein.

However, the above results are mainly on the stability of considered stochastic neural networks. And it is well known that studies on neural dynamical systems not only involve a discussion of stability properties, but also involve many dynamic behaviors such as periodic oscillatory behavior. On the other hand, the neural networks are often subject to impulsive effects that in turn affect dynamical behaviors of the systems. Moreover, a leakage delay, which is the time delay in the leakage term of the systems and a considerable factor affecting dynamics for the worse in the systems, is being put to use in the problem of stability for neural networks. However, so far, very little attention has been paid to neural networks with time delay in the leakage (or "forgetting") term. Such time delays in the leakage term are difficult to handle but have great impact on the dynamical behavior of neural networks. Therefore, it is meaningful to consider neural networks with time delays in the leakage term [24-32].

But to the best of our knowledge, there are few papers published on studying the existence of periodic solutions of impulsive stochastic neural networks with time delay in the leakage term. Motivated by the previous discussions, in this paper, we consider the following impulsive stochastic BAM neural networks:

$$
\begin{aligned}
& d x_{i}(t)=\left[-a_{i}(t) x_{i}\left(t-\tau_{i}(t)\right)\right. \\
& \left.+\sum_{j=1}^{m} c_{i j}(t) f_{j}\left(y_{j}(t)\right)+I_{i}(t)\right] d t
\end{aligned}
$$




$$
\begin{gathered}
+\sum_{j=1}^{m} \sigma_{1 i j}\left(y_{j}(t)\right) d w_{1 j}(t), \\
t \geq t_{0}, \quad t \neq t_{k}, \\
x_{i}\left(t_{k}^{+}\right)=\alpha_{i k} x_{i}\left(t_{k}\right), \quad t>0, \quad t=t_{k}, \\
d y_{j}(t)=\left[-b_{j}(t) y_{j}\left(t-\sigma_{j}(t)\right)\right. \\
\left.+\sum_{i=1}^{n} d_{j i}(t) g_{i}\left(x_{i}(t)\right)+J_{j}(t)\right] d t \\
+\sum_{i=1}^{n} \sigma_{2 j i}\left(x_{i}(t)\right) d w_{2 i}(t), \\
t \geq t_{0}, \quad t \neq t_{k}, \\
y_{j}\left(t_{k}^{+}\right)= \\
\gamma_{j k} y_{j}\left(t_{k}\right), \quad t>0, \quad t=t_{k},
\end{gathered}
$$

where $i=1,2, \ldots, n, j=1,2, \ldots, m$ ( $n$ and $m$ are the number of neurons in layers) and $x_{i}(t)$ and $y_{j}(t)$ denote the activations of the $i$ th neuron and the $j$ th neuron at time $t$; $a_{i}$ and $b_{j}$ represent the rate with which the $i$ th neuron and $j$ th neuron will reset their potential to the resting state in isolation when they are disconnected from the network and the external inputs at time $t ; 0<\tau_{i}<\bar{\tau}_{i}$ and $0<\sigma_{j}<\bar{\sigma}_{j}$ denote the leakage delays; $f_{j}$ and $g_{i}$ are the input-output functions (the activation functions); $c_{i j}$ and $d_{j i}$ are elements of feedback templates at time $t ; I_{i}$ and $J_{j}$ denote biases of the $i$ th neuron and the $j$ th neuron at time $t, i=1,2, \ldots, n, j=$ $1,2, \ldots, m ; w_{1}(t)=\left(w_{11}(t), \ldots, w_{1 m}(t)\right)^{T}$ and $w_{2}(t)=$ $\left(w_{21}(t), \ldots, w_{2 n}(t)\right)^{T}$ are $n$-dimensional Brownian motions defined on complete probability space $\left(\Omega, F,\left\{F_{t}\right\}_{t>0}, P\right)$; here, we denote by $F$ the associated $\sigma$-algebra generated by $\left\{w_{k}(t)\right\}$ with the probability measure $P, k=1,2 ; \sigma_{1 i j}$ and $\sigma_{2 j i}$ are Borel measurable functions; $\sigma_{1}=\left(\sigma_{1 i j}\right)_{n \times m}$ and $\sigma_{2}=$ $\left(\sigma_{2 j i}\right)_{m \times m}$ are diffusion coefficient matrices; $x_{i}\left(t_{k}^{+}\right)=\alpha_{i k} x_{i}\left(t_{k}\right)$ and $y_{j}\left(t_{k}^{+}\right)=\gamma_{j k} y_{j}\left(t_{k}\right)$ are impulses at moment $t$, which describe that the evolution processes experience abrupt change of state at the moments of time $t_{k}$, where $t_{0}<t_{1}<$ $t_{2}<\cdots$ and $\lim _{k \rightarrow \infty} t_{k}=\infty$.

Our main purpose in this paper is using an integral inequality, which is from a lemma in [33], to establish some sufficient conditions on the existence and $p$-exponential stability of the periodic solutions of (1).

Let $\left(\Omega, F,\left\{F_{t}\right\}_{t>0}, P\right)$ be a complete probability space with a filtration $\left\{F_{t}\right\}_{t>0}$ satisfying the usual conditions; that is, $\left\{F_{t}\right\}_{t>0}$ is right continuous and $F_{0}$ contains all $P$-null sets. Denote by $\mathrm{PC}_{F_{0}}^{b}\left(R, R^{n+m}\right)$ the family of $F_{0}$-measurable, $R^{n}$ valued random variables $z(t)$, where $z(t)$ is an piecewisecontinuous stochastic process; that is, $z(s)$ is continuous for all but at most countable points $t \in R$ and at these points $s \in$ $R, z\left(s^{+}\right)$and $z\left(s^{-}\right)$exist, $z\left(s^{-}\right)=z(s)$. For $z \in \operatorname{PC}_{F_{0}}^{b}\left(R, R^{n+m}\right)$, define the norm $\|z\|=\max _{0 \leq t \leq \omega}\left(E|z(t)|_{1}^{p}\right)^{1 / p}$, where $p>1$ is an integer, $|z(t)|_{1}=\left(\sum_{i=1}^{n+m}\left|z_{i}(t)\right|^{2}\right)^{1 / 2} ; E(\cdot)$ stands for the correspondent expectation operator with respect to the given probability measure $P$.

For convenience, for an $\omega$-periodic function $f: R \rightarrow R$, denote $\bar{f}=\max _{0 \leq t \leq \omega}|f(t)|, \underline{f}=\min _{0 \leq t \leq \omega}|f(t)|$. The initial condition of (1) is

$$
x_{i}(s)=\varphi_{i}(s), \quad y_{j}(s)=\psi_{j}(s), \quad s \leq t_{0},
$$

where $\varphi_{i}, \psi_{j} \quad \in \quad \mathrm{PC}_{F_{0}}^{b}\left(\left(-\delta, t_{0}\right], R\right), \quad \delta=$ $\max \left\{\max _{1 \leq i \leq n} \bar{\tau}_{i}, \max _{1 \leq j \leq m} \bar{\sigma}_{j}\right\}, i=1,2, \ldots, n, j=$ $1,2, \ldots, m$.

Throughout this paper, we assume that the following conditions hold:

$\left(H_{1}\right) a_{i}(t)>0, b_{j}(t)>0, \tau_{i}(t), \sigma_{j}(t), c_{i j}(t), d_{j i}(t), I_{i}(t)$, and $J_{j}(t)$ are all periodic continuous functions with period $\omega$ for $t \in R, i=1,2, \ldots, n, j=1,2, \ldots, m$;

$\left(H_{2}\right) f_{j}, g_{i}, \sigma_{1 i j}$, and $\sigma_{2 j i}$ are Lipschitz-continuous with Lipschitz constants $L_{j}^{f}>0, L_{i}^{g}>0, l_{1 i j}>0, l_{2 j i}>0$ and $f_{j}(0)=g_{i}(0)=\sigma_{1 i j}(0)=\sigma_{2 j i}(0)=0, i=$ $1,2, \ldots, n, j=1,2, \ldots, m$;

$\left(H_{3}\right)\left\{\alpha_{i k}\right\}$ and $\left\{\gamma_{j k}\right\}$ are real sequences and $\prod_{t_{0}<t_{k}<t} \alpha_{i k}$ and $\prod_{t_{0}<t_{k}<t} \gamma_{j k}$ are $\omega$-periodic, $i=1,2, \ldots, n, j=$ $1,2, \ldots, m, k=1,2, \ldots$

Remark 1. From $\left(H_{3}\right)$, there exist constants $\bar{\alpha}, \underline{\alpha}, \bar{\gamma}$, and $\gamma$ such that

$$
\begin{aligned}
& 0 \leq \underline{\alpha} \leq\left|\prod_{t_{0}<t_{k}<t} \alpha_{i k}\right| \leq \bar{\alpha}, \\
& 0 \leq \underline{\gamma} \leq\left|\prod_{t_{0}<t_{k}<t} \gamma_{j k}\right| \leq \bar{\gamma},
\end{aligned}
$$

where $i=1,2, \ldots, n, j=1,2, \ldots, m$, and $k=1,2, \ldots$.

This paper is organized as follows. In Section 2, we introduce some definitions and state some preliminary results which are needed in later sections. In Section 3, we state and prove our results. In Section 4, we give an example to illustrate the feasibility of our results obtained in the previous section.

\section{Preliminaries}

In this section, we introduce some definitions and state some preliminary results.

Definition 2. A stochastic process $x_{t}(s)$ is said to be periodic with period $\omega$ if its finite dimensional distributions are periodic with period $\omega$; that is, for any positive integer $m$ and any moments of time $t_{1}, t_{2}, \ldots, t_{m}$, the joint distribution of the random variables $x_{t_{1}+k \omega}(s), x_{t_{2}+k \omega}(s), \ldots, x_{t_{m}+k \omega}(s)$ is independent of $k, k= \pm 1, \pm 2, \ldots$ 
Lemma 3 (see [34]). If $x(t)$ is an $\omega$-periodic stochastic process, then its mathematical expectation and variance are $\omega$-periodic.

Definition 4. The solution $x_{t}\left(t_{0}, \varphi\right)$ of (1) is said to be

(i) $p$-uniformly bounded if for each $\alpha>0, t_{0} \in R$, there exists a positive constant $\theta=\theta(\alpha)$ which is independent of $t_{0}$ such that $\|\varphi\|^{p} \leq \alpha$ implies $E\left(\left\|x_{t}\left(t_{0}, \varphi\right)\right\|^{p}\right) \leq \theta, t \geq t_{0}$,

(ii) $p$-point dissipative if there exists a constant $N>0$ such that for any point $\varphi \in \operatorname{PC}_{F_{0}}^{b}\left([-\tau, 0], R^{n}\right)$, there exists $T\left(t_{0}, \varphi\right)$ such that for each $\alpha>0, t_{0} \in R$, there exists a positive constant $\theta=\theta(\alpha)$ which is independent of $t_{0}$ such that $E\left(\left\|x_{t}\left(t_{0}, \varphi\right)\right\|^{p}\right) \leq N, t \geq$ $t_{0}+T\left(t_{0}, \varphi\right)$.

Lemma 5 (see [35]). Under conditions $\left(H_{1}\right)-\left(H_{3}\right)$, assume that the solution of (1) is p-uniformly bounded and p-point dissipative for $p>2$; then (1) has an w-periodic solution.

Lemma 6 (see [36]). For any $x \in R_{+}^{n}$ and $p>0$,

$$
\begin{aligned}
& |x|^{p} \leq n^{((p / 2)-1) \vee 0} \sum_{i=1}^{n} x_{i}^{p}, \\
& \left(\sum_{i=1}^{n} x_{i}\right)^{p} \leq n^{(p-1) \vee 0} \sum_{i=1}^{n} x_{i}^{p} .
\end{aligned}
$$

Definition 7. The periodic solution $x\left(t, t_{0}, \varphi\right)$ with initial value $\varphi \in \mathrm{PC}_{F_{0}}^{b}\left([-\tau, 0], R^{n}\right)$ of $(1)$ is said to be $p$-exponential stable if there are constants $\lambda>0$ and $M>1$ such that any solution $y\left(t, t_{0}, \varphi_{1}\right)$ with initial value $\varphi_{1} \in \mathrm{PC}_{F_{0}}^{b}\left([-\tau, 0], R^{n}\right)$ of $(1)$ satisfies

$$
E\left(|x-y|_{1}^{p}\right) \leq M\left\|\varphi-\varphi_{1}\right\|^{p} \mathrm{e}^{-\lambda\left(t-t_{0}\right)}, \quad t \geq t_{0} .
$$

Lemma 8 (see [33]). Let $u(t) \in C\left(R, R_{+}^{n}\right)$ be a solution of the delay integral inequality

$$
\begin{aligned}
& u(t) \leq M_{1} \mathrm{e}^{-\delta\left(t-t_{0}\right)}[\varphi]_{\tau}^{+} \\
&+\int_{t_{0}}^{t} \mathrm{e}^{-C_{1}(t-s)} A_{1} u(s) d s \\
&+\int_{t_{0}}^{t} \mathrm{e}^{-C_{1}(t-s)} B_{1}[u(s)]_{\tau}^{+} d s+J_{1}, \\
& u(t) \leq \varphi(t), \quad \forall t \in\left[t_{0}-\tau, t_{0}\right],
\end{aligned}
$$

where $A_{1}, B_{1}, C_{1}$, and $M_{1} \in R_{+}^{n \times n}, J_{1} \geq 0$ is a constant vector, and $\varphi(t) \in C\left(\left[t_{0}-\tau, t_{0}\right], R_{+}^{n}\right)$. If $\rho\left(\Pi=C_{1}^{-1}\left(A_{1}+B_{1}\right)\right)<1$, then there are constants $0<\lambda \leq \delta$ and $N \geq 1$ such that

$$
u(t) \leq N z \mathrm{e}^{-\lambda\left(t-t_{0}\right)}+(I-\Pi)^{-1} J_{1}, \quad t \geq t_{0},
$$

where $z$ satisfies $[\varphi]_{\tau}^{+} \leq z$.
Lemma 9 (see [33]). Assume that all conditions of Lemma 9 hold. If $J_{1}=0$, then all solutions of inequality of (6) exponentially converge to zero.

By Lemmas 8 and 9, we have the following corollary.

Corollary 10. Let $u(t) \in C\left(R, R_{+}\right)$be a solution of the delay integral inequality

$$
\begin{aligned}
& u(t) \leq M_{1} \mathrm{e}^{-\delta\left(t-t_{0}\right)}[\varphi]_{\tau}^{+} \\
&+\int_{t_{0}}^{t} \mathrm{e}^{-C_{1}(t-s)} A_{1} u(s) d s \\
&+\int_{t_{0}}^{t} \mathrm{e}^{-C_{1}(t-s)} B_{1}[u(s)]_{\tau}^{+} d s+J_{1}, \\
& t \geq t_{0}, \\
& u(t) \leq \varphi(t), \quad \forall t \in\left[t_{0}-\tau, t_{0}\right],
\end{aligned}
$$

where $A_{1}, B_{1}, C_{1}$, and $M_{1} \in R_{+}, J_{1} \geq 0$ is a constant, and $\varphi(t) \in C\left(\left[t_{0}-\tau, t_{0}\right], R_{+}\right)$. If $\left(A_{1}+B_{1}\right) / C_{1}<1$, then there are constants $0<\lambda \leq \delta$ and $N \geq 1$ such that

$$
\begin{array}{r}
u(t) \leq N z \mathrm{e}^{-\lambda\left(t-t_{0}\right)}+\left(1-\frac{A_{1}+B_{1}}{C_{1}}\right)^{-1} J_{1} \\
t \geq t_{0}
\end{array}
$$

where $z$ satisfies $[\varphi]_{\tau}^{+} \leq z$. Moreover, if $J_{1}=0$, then all solutions of inequality of (8) exponentially converge to zero.

Under our assumptions, we consider the following system:

$$
\begin{aligned}
d u_{i}(t)=\left[-a_{i}(t) u_{i}\left(t-\tau_{i}(t)\right)\right. \\
+\prod_{t_{0}<t_{k}<t} \alpha_{i k}^{-1} \sum_{j=1}^{m} c_{i j}(t) f_{j}\left(\prod_{t_{0}<t_{k}<t} \gamma_{j k} v_{j}(t)\right) \\
\left.+\prod_{t_{0}<t_{k}<t} \alpha_{i k}^{-1} I_{i}(t)\right] d t \\
+\prod_{t_{0}<t_{k}<t} \alpha_{i k}^{-1} \sum_{j=1}^{m} \sigma_{1 i j}\left(\prod_{t_{0}<t_{k}<t} \gamma_{j k} v_{j}(t)\right) d w_{1 j}(t),
\end{aligned}
$$




$$
\begin{aligned}
& d v_{j}(t)=\left[-b_{j}(t) v_{j}\left(t-\sigma_{j}(t)\right)\right. \\
& \epsilon_{1}=6^{p-1} \sum_{i=1}^{n}\left[\underline{a}_{i}^{1-p}\left(\frac{\bar{\gamma}}{\underline{\alpha}} \sum_{j=1}^{m}\left(\bar{c}_{i j} L_{j}^{f}\right)^{p /(p-1)}\right)^{p-1}\right. \\
& +\prod_{t_{0}<t_{k}<t} \gamma_{j k}^{-1} \sum_{i=1}^{n} d_{j i}(t) g_{i}\left(\prod_{t_{0}<t_{k}<t} \alpha_{i k} u_{i}(t)\right) \\
& \left.+\prod_{t_{0}<t_{k}<t} \gamma_{j k}^{-1} J_{j}(t)\right] d t \\
& +\prod_{t_{0}<t_{k}<t} \gamma_{j k}^{-1} \sum_{i=1}^{n} \sigma_{2 j i}\left(\prod_{t_{0}<t_{k}<t} \alpha_{i k} u_{i}(t)\right) d w_{2 i}(t), \\
& t \geq t_{0} \text {, } \\
& +l_{p} n^{(p / 2)-1}\left(\frac{2 \underline{a}_{i}(p-1)}{p-2}\right)^{1-(p / 2)} \\
& \left.\times\left(\frac{\bar{\gamma}}{\underline{\alpha}} \sum_{j=1}^{m}\left(l_{1 i j}\right)^{p /(p-1)}\right)^{p-1}\right] \\
& \epsilon_{2}=6^{p-1} \sum_{j=1}^{m}\left[\underline{b}_{j}^{1-p}\left(\frac{\bar{\alpha}}{\underline{\gamma}} \sum_{i=1}^{n}\left(\bar{d}_{j i} L_{i}^{g}\right)^{p /(p-1)}\right)^{p-1}\right.
\end{aligned}
$$
lemma.

Lemma 11. Let $\left(H_{1}\right)-\left(H_{3}\right)$ hold. Then,

(i) if $\left(u_{1}(t), \ldots, u_{n}(t), v_{1}(t), \ldots, v_{m}(t)\right)^{T}$ is a solution of (10), then $\left(\prod_{t_{0}<t_{k}<t} \alpha_{1 k} u_{1}(t), \ldots, \prod_{t_{0}<t_{k}<t} \alpha_{n k} u_{n}(t)\right.$, $\left.\prod_{t_{0}<t_{k}<t} \gamma_{1 k} v_{1}(t), \ldots, \prod_{t_{0}<t_{k}<t} \gamma_{m k} v_{m}(t)\right)^{T}$ is a solution of (1);

(ii) if $\left(x_{1}(t), \ldots, x_{n}(t), y_{1}(t), \ldots, y_{m}(t)\right)^{T}$ is a solution of (1), then $\left(\prod_{t_{0}<t_{k}<t} \alpha_{i k}^{-1} x_{1}(t), \ldots, \prod_{t_{0}<t_{k}<t} \alpha_{i k}^{-1} x_{n}(t)\right.$, $\left.\prod_{t_{0}<t_{k}<t} \gamma_{j k}^{-1} y_{1}(t), \ldots, \prod_{t_{0}<t_{k}<t} \gamma_{m k}^{-1} y_{m}(t)\right)^{T}$ is a solution of (10).

\section{Main Results}

In this section, we will state and prove the sufficient conditions for the existence and $p$-exponential stability of periodic solution of (1).

Theorem 12. Let $\left(H_{1}\right)-\left(H_{3}\right)$ hold. Suppose further that

$\left(H_{4}\right)$ there exists an integer $p>2$ such that $(\epsilon+c) / \theta<1$, where

$$
\begin{gathered}
\theta=\min \left\{\min _{1 \leq i \leq n} \underline{a}_{i}, \min _{1 \leq j \leq m} \underline{b}_{j}\right\}, \\
c=6^{p-1} \max \left\{\max _{1 \leq i \leq n} \bar{a}_{i}^{p}, \max _{1 \leq j \leq m} \bar{b}_{j}^{p}\right\}, \\
\epsilon=\max \left\{c, \epsilon_{1}, \epsilon_{2}\right\},
\end{gathered}
$$

Then (1) has an w-periodic solution, which is pexponentially stable.

Proof. We can rewrite (10) as follows:

$$
\begin{aligned}
d u_{i}(t)= & {\left[-a_{i}(t) u_{i}(t)+a_{i}(t) u_{i}(t)\right.} \\
& -a_{i}(t) u_{i}\left(t-\tau_{i}(t)\right)+\prod_{t_{0}<t_{k}<t} \alpha_{i k}^{-1} \sum_{j=1}^{m} c_{i j}(t) f_{j} \\
& \left.\times\left(\prod_{t_{0}<t_{k}<t} \gamma_{j k} v_{j}(t)\right)+\prod_{t_{0}<t_{k}<t} \alpha_{i k}^{-1} I_{i}(t)\right] d t \\
& +\prod_{t_{0}<t_{k}<t} \alpha_{i k}^{-1} \sum_{j=1}^{m} \sigma_{1 i j}\left(\prod_{t_{0}<t_{k}<t} \gamma_{j k} v_{j}(t)\right) d w_{1 j}(t), \\
& \\
& \left.\left.+\prod_{t_{0}<t_{k}<t} \alpha_{i k} u_{i}(t)\right)+\prod_{t_{0}<t_{k}<t} \gamma_{j k}^{-1} J_{j}(t)\right] d t \\
& -b_{j}(t) v_{j}\left(t-\sigma_{j}(t)\right)+\prod_{t_{0}<t_{k}<t} \gamma_{j k}^{-1} \sum_{i=1}^{n} d_{j i}(t) g_{i}, n,
\end{aligned}
$$




$$
\begin{array}{r}
+\prod_{t_{0}<t_{k}<t} \gamma_{j k}^{-1} \sum_{i=1}^{n} \sigma_{2 j i}\left(\prod_{t_{0}<t_{k}<t} \alpha_{i k} u_{i}(t)\right) d w_{2 i}(t), \\
t \geq t_{0}, \quad j=1,2, \ldots, m .
\end{array}
$$

By the method of variation parameter, for $t \geq t_{0}, i=$ $1,2, \ldots, n$, from the first equation of (12), we have the following:

$$
\begin{aligned}
u_{i}(t)= & u_{i}\left(t_{0}\right) \mathrm{e}^{-\int_{t_{0}}^{t} a_{i}(9) d \vartheta} \\
+ & \int_{t_{0}}^{t} \mathrm{e}^{-\int_{s}^{t} a_{i}(9) d \vartheta} \\
& \times\left[a_{i}(s) u_{i}(s)-a_{i}(s) u_{i}\left(s-\tau_{i}(s)\right)\right. \\
& +\prod_{t_{0}<t_{k}<t} \alpha_{i k}^{-1} \sum_{j=1}^{m} c_{i j}(s) f_{j} \\
& \left.\times\left(\prod_{t_{0}<t_{k}<t} \gamma_{j k} v_{j}(s)\right)+\prod_{t_{0}<t_{k}<t} \alpha_{i k}^{-1} I_{i}(s)\right] d s \\
& \int_{t_{0}}^{t} \mathrm{e}^{-\int_{s}^{t} a_{i}(9) d 9} \prod_{t_{0}<t_{k}<t} \alpha_{i k}^{-1} \sum_{j=1}^{m} \sigma_{1 i j} \\
\times & \left(\prod_{t_{0}<t_{k}<t} \gamma_{j k} v_{j}(s)\right) d w_{1 j}(s) .
\end{aligned}
$$

For $i=1,2, \ldots, n$, denote

$$
\begin{gathered}
F_{1 i}=u_{i}\left(t_{0}\right) \mathrm{e}^{-\int_{t_{0}}^{t} a_{i}(9) d \vartheta}, \\
F_{2 i}=\int_{t_{0}}^{t} \mathrm{e}^{-\int_{s}^{t} a_{i}(9) d \vartheta} a_{i}(s) u_{i}(s) d s, \\
F_{3 i}=\int_{t_{0}}^{t} \mathrm{e}^{-\int_{s}^{t} a_{i}(9) d \vartheta} a_{i}(s) u_{i}\left(s-\tau_{i}(s)\right) d s, \\
F_{4 i}=\int_{t_{0}}^{t} \mathrm{e}^{-\int_{s}^{t} a_{i}(9) d \vartheta} \prod_{t_{0}<t_{k}<t} \alpha_{i k}^{-1} I_{i}(s) d s, \\
F_{5 i}=\int_{t_{0}}^{t} \mathrm{e}^{-\int_{s}^{t} a_{i}(9) d \vartheta} \prod_{t_{0}<t_{k}<t} \alpha_{i k}^{-1} \\
\times \sum_{j=1}^{m} c_{i j}(s) f_{j}\left(\prod_{t_{0}<t_{k}<t} \gamma_{j k} v_{j}(s)\right) d s,
\end{gathered}
$$

$$
\begin{aligned}
F_{6 i}=\int_{t_{0}}^{t} \mathrm{e}^{-\int_{s}^{t} a_{i}(9) d \vartheta} \prod_{t_{0}<t_{k}<t} \alpha_{i k}^{-1} \\
\quad \times \sum_{j=1}^{m} \sigma_{1 i j}\left(\prod_{t_{0}<t_{k}<t} \gamma_{j k} v_{j}(s)\right) d w_{1 j}(s) .
\end{aligned}
$$

Considering expectations, using Lemma 6 , for $i=1,2, \ldots, n$, we have

$$
\begin{aligned}
E\left|u_{i}(t)\right|^{p} \leq 6^{p-1} E & \left(\left|F_{1 i}\right|^{p}+\left|F_{2 i}\right|^{p}+\left|F_{3 i}\right|^{p}\right. \\
& \left.+\left|F_{4 i}\right|^{p}+\left|F_{5 i}\right|^{p}+\left|F_{6 i}\right|^{p}\right) .
\end{aligned}
$$

For $i=1,2, \ldots, n$, we evaluate the first term of (15) as follows:

$$
\begin{aligned}
E\left|F_{1 i}\right|^{p} & =E\left|u_{i}\left(t_{0}\right) \mathrm{e}^{-\int_{t_{0}}^{t} a_{i}(9) d \vartheta}\right|^{p} \\
& \leq E\left|u_{i}\left(t_{0}\right) \mathrm{e}^{-\underline{a}_{i}\left(t-t_{0}\right)}\right|^{p} \\
& \leq \mathrm{e}^{-p \underline{a}_{i}\left(t-t_{0}\right)} E\left|u_{i}\left(t_{0}\right)\right|^{p} .
\end{aligned}
$$

For the second term of (15), we have

$$
\begin{aligned}
& E\left|F_{2 i}\right|^{p}=E\left|\int_{t_{0}}^{t} \mathrm{e}^{-\int_{s}^{t} a_{i}(9) d \vartheta} a_{i}(s) u_{i}(s) d s\right|^{p} \\
& \leq E\left(\int_{t_{0}}^{t} \mathrm{e}^{-\int_{s}^{t} a_{i}(9) d \vartheta}\left|a_{i}(s)\right|\left|u_{i}(s)\right| d s\right)^{p} \\
& \leq E\left(\int_{t_{0}}^{t} \bar{a}_{i} \mathrm{e}^{-\underline{a}_{i}(t-s)}\left|u_{i}(s)\right| d s\right)^{p} \\
&=\bar{a}_{i}^{p} \int_{t_{0}}^{t} \mathrm{e}^{-p \underline{a}_{i}(t-s)} E\left|u_{i}(s)\right|^{p} d s, \\
& i=1,2, \ldots, n .
\end{aligned}
$$

For the third term of (15), we have

$$
\begin{aligned}
E\left|F_{3 i}\right|^{p} & =E\left|\int_{t_{0}}^{t} \mathrm{e}^{-\int_{s}^{t} a_{i}(9) d \vartheta} a_{i}(s) u_{i}\left(s-\tau_{i}(s)\right) d s\right|^{p} \\
& \leq E\left(\int_{t_{0}}^{t} \mathrm{e}^{-\int_{s}^{t} a_{i}(\vartheta) d \vartheta}\left|a_{i}(s)\right|\left|u_{i}\left(s-\tau_{i}(s)\right)\right| d s\right)^{p} \\
& \leq E\left(\int_{t_{0}}^{t} \bar{a}_{i} \mathrm{e}^{-\underline{a}_{i}(t-s)}\left|u_{i}\left(s-\tau_{i}(s)\right)\right| d s\right)^{p}
\end{aligned}
$$




$$
\begin{array}{r}
=\bar{a}_{i}^{p} \int_{t_{0}}^{t} \mathrm{e}^{-p \underline{a}_{i}(t-s)} E\left|u_{i}\left(s-\tau_{i}(s)\right)\right|^{p} d s \\
i=1,2, \ldots, n .
\end{array}
$$

As for the fourth term of (15), for $i=1,2, \ldots, n$, we have

$$
\begin{aligned}
E\left|F_{4 i}\right|^{p} & =E\left|\int_{t_{0}}^{t} \mathrm{e}^{-\int_{s}^{t} a_{i}(\vartheta) d \vartheta} \prod_{t_{0}<t_{k}<t} \alpha_{i k}^{-1} I_{i}(s) d s\right|^{p} \\
& \leq E\left|\int_{t_{0}}^{t} \mathrm{e}^{-\underline{a}_{i}(t-s)} \prod_{t_{0}<t_{k}<t} \alpha_{i k}^{-1} I_{i}(s) d s\right|^{p} \\
& \leq\left(\frac{\bar{I}_{i}}{\underline{a}_{i} \underline{\alpha}}\right)^{p} .
\end{aligned}
$$

For the fifth term of (15), by Hölder inequality, we have

$$
\begin{aligned}
& E\left|F_{5 i}\right|^{p}=E \mid \int_{t_{0}}^{t} \mathrm{e}^{-\int_{s}^{t} a_{i}(\vartheta) d \vartheta} \prod_{t_{0}<t_{k}<t} \alpha_{i k}^{-1} \\
& \times\left.\sum_{j=1}^{m} c_{i j}(s) f_{j}\left(\prod_{t_{0}<t_{k}<t} \gamma_{j k} v_{j}(s)\right) d s\right|^{p} \\
& \leq E\left(\int_{t_{0}}^{t} \mathrm{e}^{-\underline{a}_{i}(t-s)}\left|\prod_{t_{0}<t_{k}<t} \alpha_{i k}^{-1}\right| \sum_{j=1}^{m}\left|c_{i j}(s)\right|\right. \\
& \left.\times\left|f_{j}\left(\prod_{t_{0}<t_{k}<t} \gamma_{j k} v_{j}(s)\right)\right| d s\right)^{p} \\
& \leq E\left(\int_{t_{0}}^{t} \mathrm{e}^{-\underline{a}_{i}(t-s)}\right. \\
& \times\left|\prod_{t_{0}<t_{k}<t} \alpha_{i k}^{-1}\right| \sum_{j=1}^{m}\left|c_{i j}(s)\right| L_{j}^{f} \\
& \left.\times\left|\prod_{t_{0}<t_{k}<t} \gamma_{j k} v_{j}(s)\right| d s\right)^{p} \\
& \leq E\left(\int_{t_{0}}^{t} \mathrm{e}^{-\underline{a}_{i}(t-s)} \frac{\bar{\gamma}}{\underline{\alpha}} \sum_{j=1}^{m}\left|c_{i j}(s)\right|\right. \\
& \left.\times L_{j}^{f}\left|v_{j}(s)\right| d s\right)^{p}
\end{aligned}
$$

$$
\begin{array}{r}
=E\left(\int_{t_{0}}^{t}\left(\mathrm{e}^{-\underline{a}_{i}(t-s)}\right)^{(p-1) / p}\left(\mathrm{e}^{-\underline{a}_{i}(t-s)}\right)^{1 / p}\right. \\
\left.\times \frac{\bar{\gamma}}{\underline{\alpha}_{j=1}^{m}}\left|c_{i j}(s)\right| L_{j}^{f}\left|v_{j}(s)\right| d s\right)^{p}
\end{array}
$$

$$
\begin{gathered}
\leq E\left(\left(\int_{t_{0}}^{t} \mathrm{e}^{-\underline{a}_{i}(t-s)} d s\right)^{p-1}\right. \\
\left.\quad \times \int_{t_{0}}^{t} \mathrm{e}^{-\underline{\underline{a}}_{i}(t-s)}\left(\frac{\bar{\gamma}}{\underline{\alpha}} \sum_{j=1}^{m} \bar{c}_{i j} L_{j}^{f}\left|v_{j}(s)\right|\right)^{p} d s\right) \\
\leq \underline{a}_{i}^{1-p} \int_{t_{0}}^{t} \mathrm{e}^{-\underline{a}_{i}(t-s)}\left(\frac{\bar{\gamma}}{\underline{\alpha}} \sum_{j=1}^{m}\left(\bar{c}_{i j} L_{j}^{f}\right)^{p /(p-1)}\right)^{p-1} \\
\quad \times \sum_{j=1}^{m} E\left|v_{j}(s)\right|^{p} d s, \quad i=1,2, \ldots, n .
\end{gathered}
$$

As for the last term of (15), using Proposition 1.9 in [37] and Hölder inequality, for $i=1,2, \ldots, n$, we have

$$
\begin{aligned}
& E\left|F_{6 i}\right|^{p}=E \mid \int_{t_{0}}^{t} \mathrm{e}^{-\int_{s}^{t} a_{i}(9) d \vartheta} \prod_{t_{0}<t_{k}<t} \alpha_{i k}^{-1} \\
& \times\left.\sum_{j=1}^{m} \sigma_{1 i j}\left(\prod_{t_{0}<t_{k}<t} \gamma_{j k} v_{j}(s)\right) d w_{1 j}(s)\right|^{p} \\
& \leq l_{p}\left[\int _ { t _ { 0 } } ^ { t } \left(\mathrm{e}^{-p \underline{a}_{i}(t-s)} E \mid \prod_{t_{0}<t_{k}<t} \alpha_{i k}^{-2} \sum_{j=1}^{n} \sigma_{1 i j}^{2}\right.\right. \\
& \left.\left.\times\left.\left(\prod_{t_{0}<t_{k}<t} \gamma_{j k} v_{j}(s)\right)\right|^{p / 2}\right)^{2 / p} d s\right]^{p / 2} \\
& \leq l_{p} n^{(p / 2)-1}\left[\int _ { t _ { 0 } } ^ { t } \left(\mathrm{e}^{-p \underline{a}_{i}(t-s)}\right.\right. \\
& \left.\left.\times E\left(\frac{\bar{\gamma}}{\underline{\alpha}} \sum_{j=1}^{n} l_{1 i j}\left|v_{j}(s)\right|\right)^{p}\right)^{2 / p} d s\right]^{p / 2} \\
& =l_{p} n^{(p / 2)-1}\left[\int _ { t _ { 0 } } ^ { t } \left(\mathrm{e}^{-(p-1) \underline{a}_{i}(t-s)} \mathrm{e}^{-\underline{a}_{i}(t-s)}\right.\right. \\
& \left.\left.\times E\left(\frac{\bar{\gamma}}{\underline{\alpha}} \sum_{j=1}^{n} l_{1 i j}\left|v_{j}(s)\right|\right)^{p}\right)^{2 / p} d s\right]^{p / 2}
\end{aligned}
$$




$$
\begin{aligned}
\leq & l_{p} n^{(p / 2)-1}\left(\int_{t_{0}}^{t} \mathrm{e}^{\left(-2 \underline{a}_{i}(p-1) /(p-2)\right)(t-s)} d s\right)^{(p / 2)-1} \\
& \times\left(\int_{t_{0}}^{t} \mathrm{e}^{-\underline{a}_{i}(t-s)} E\left(\frac{\bar{\gamma}}{\underline{\alpha}} \sum_{j=1}^{n} l_{1 i j}\left|v_{j}(s)\right|\right)^{p} d s\right) \\
\leq & l_{p} n^{(p / 2)-1}\left(\frac{2 \underline{a}_{i}(p-1)}{p-2}\right)^{1-(p / 2)} \\
& \times\left(\int_{t_{0}}^{t} \mathrm{e}^{-\underline{a}_{i}(t-s)}\right. \\
\times & \left.\times E\left(\frac{\bar{\gamma}}{\underline{\alpha}} \sum_{j=1}^{m} l_{1 i j}\left|v_{j}(s)\right|\right)^{p} d s\right) \\
\leq & l_{p} n^{(p / 2)-1}\left(\frac{2 \underline{a}_{i}(p-1)}{p-2}\right)^{1-(p / 2)} \\
& \times\left(\int_{t_{0}}^{t} \mathrm{e}^{-\underline{a}_{i}(t-s)}\left(\frac{\bar{\gamma}}{\underline{\alpha}} \sum_{j=1}^{m}\left(l_{1 i j}\right)^{p /(p-1)}\right)^{p-1}\right. \\
& \left.\times \sum_{j=1}^{m} E\left|v_{j}(s)\right|^{p} d s\right),
\end{aligned}
$$

where $l_{p}=(p(p-1) / 2)^{p / 2}$. Therefore, for $i=1,2, \ldots, n$, we have

$E\left|u_{i}(t)\right|^{p}$

$$
\begin{aligned}
& \leq 6^{p-1}\left\{\mathrm{e}^{-p \underline{a}_{i}\left(t-t_{0}\right)} E\left|u_{i}\left(t_{0}\right)\right|^{p}\right. \\
& +\bar{a}_{i}^{p} \int_{t_{0}}^{t} \mathrm{e}^{-p \underline{a}_{i}(t-s)} E\left|u_{i}(s)\right|^{p} d s \\
& +\bar{a}_{i}^{p} \int_{t_{0}}^{t} \mathrm{e}^{-p \underline{a}_{i}(t-s)} E\left|u_{i}\left(s-\tau_{i}(s)\right)\right|^{p} d s \\
& +\underline{a}_{i}^{1-p} \int_{t_{0}}^{t} \mathrm{e}^{-\underline{a}_{i}(t-s)} \\
& \times\left(\frac{\bar{\gamma}}{\alpha} \sum_{j=1}^{m}\left(\bar{c}_{i j} L_{j}^{f}\right)^{p /(p-1)}\right)^{p-1} \\
& \times \sum_{j=1}^{n} E\left|v_{j}(s)\right|^{p} d s
\end{aligned}
$$

$$
\begin{array}{r}
+l_{p} n^{(p / 2)-1}\left(\frac{2 \underline{a}_{i}(p-1)}{p-2}\right)^{1-(p / 2)} \\
\times\left(\int_{t_{0}}^{t} \mathrm{e}^{-\underline{a}_{i}(t-s)}\left(\frac{\bar{\gamma}}{\underline{\alpha}} \sum_{j=1}^{m}\left(l_{1 i j}\right)^{p /(p-1)}\right)^{p-1}\right. \\
\left.\left.\times \sum_{j=1}^{m} E\left|v_{j}(s)\right|^{p} d s\right)+\left(\frac{\bar{I}_{i}}{\underline{a}_{i} \underline{\alpha}}\right)^{p}\right\}
\end{array}
$$$$
=6^{p-1}\left\{\mathrm{e}^{-p \underline{a}_{i}\left(t-t_{0}\right)} E\left|u_{i}\left(t_{0}\right)\right|^{p}\right.
$$$$
+\bar{a}_{i}^{p} \int_{t_{0}}^{t} \mathrm{e}^{-p \underline{a}_{i}(t-s)} E\left|u_{i}(s)\right|^{p} d s
$$$$
+\bar{a}_{i}^{p} \int_{t_{0}}^{t} \mathrm{e}^{-p \underline{a}_{i}(t-s)} E\left|u_{i}\left(s-\tau_{i}(s)\right)\right|^{p} d s
$$$$
+\int_{t_{0}}^{t} \mathrm{e}^{-\underline{a}_{i}(t-s)}
$$$$
\times\left[\underline{a}_{i}^{1-p}\left(\frac{\bar{\gamma}}{\underline{\alpha}} \sum_{j=1}^{m}\left(\bar{c}_{i j} L_{j}^{f}\right)^{p /(p-1)}\right)^{p-1}\right.
$$$$
+l_{p} n^{(p / 2)-1}\left(\frac{2 \underline{a}_{i}(p-1)}{p-2}\right)^{1-(p / 2)}
$$$$
\left.\times\left(\frac{\bar{\gamma}}{\underline{\alpha}} \sum_{j=1}^{m}\left(l_{1 i j}\right)^{p /(p-1)}\right)^{p-1}\right]
$$

$$
\begin{gathered}
\left.\quad \times \sum_{j=1}^{m} E\left|v_{j}(s)\right|^{p} d s+\left(\frac{\bar{I}_{i}}{\underline{a}_{i} \underline{\alpha}}\right)^{p}\right\} \\
\leq \sigma^{p-1}\left\{\mathrm{e}^{-\underline{a}_{i}\left(t-t_{0}\right)} E\left|u_{i}\left(t_{0}\right)\right|^{p}\right. \\
+\bar{a}_{i}^{p} \int_{t_{0}}^{t} \mathrm{e}^{-\underline{a}_{i}(t-s)} E\left|u_{i}(s)\right|^{p} d s \\
+\bar{a}_{i}^{p} \int_{t_{0}}^{t} \mathrm{e}^{-\underline{a}_{i}(t-s)} E\left|u_{i}\left(s-\tau_{i}(s)\right)\right|^{p} d s \\
+\int_{t_{0}}^{t} \mathrm{e}^{-\underline{a}_{i}(t-s)} \\
\times\left[\underline{a}_{i}^{1-p}\left(\frac{\bar{\gamma}}{\underline{\alpha}} \sum_{j=1}^{m}\left(\bar{c}_{i j} L_{j}^{f}\right)^{p /(p-1)}\right)^{p-1}\right. \\
\quad+l_{p} n^{(p / 2)-1}\left(\frac{2 \underline{a}_{i}(p-1)}{p-2}\right)^{1-(p / 2)}
\end{gathered}
$$




$$
\begin{array}{r}
\left.\times\left(\frac{\bar{\gamma}}{\underline{\alpha}} \sum_{j=1}^{m}\left(l_{1 i j}\right)^{p /(p-1)}\right)^{p-1}\right] \\
\left.\times \sum_{j=1}^{m} E\left|v_{j}(s)\right|^{p} d s+\left(\frac{\bar{I}_{i}}{\underline{a}_{i} \underline{\alpha}}\right)^{p}\right\} .
\end{array}
$$

Similarly, for $t \geq t_{0}, j=1,2, \ldots, m$, from the second equation of (12), we can obtain the following:

$$
\begin{aligned}
& E\left|v_{j}(t)\right|^{p} \leq 6^{p-1}\left\{\mathrm{e}^{-\underline{b}_{j}\left(t-t_{0}\right)} E\left|v_{j}\left(t_{0}\right)\right|^{p}\right. \\
&+\bar{b}_{j}^{p} \int_{t_{0}}^{t} \mathrm{e}^{-\underline{b}_{j}(t-s)} E\left|v_{j}(s)\right|^{p} d s \\
&+\bar{b}_{j}^{p} \int_{t_{0}}^{t} \mathrm{e}^{-\underline{b}_{j}(t-s)} E\left|v_{j}\left(s-\sigma_{j}(s)\right)\right|^{p} d s \\
&+\int_{t_{0}}^{t} \mathrm{e}^{-\underline{b}_{j}(t-s)} \\
& \times {\left[\underline{b}_{j}^{1-p}\left(\frac{\bar{\alpha}}{\underline{\underline{\gamma}}} \sum_{i=1}^{n}\left(\bar{d}_{j i} L_{i}^{g}\right)^{p /(p-1)}\right)^{p-1}\right.} \\
&+l_{p} m^{(p / 2)-1}\left(\frac{2 \underline{b}_{j}(p-1)}{p-2}\right)^{1-(p / 2)} \\
&\left.\times\left(\frac{\bar{\alpha}}{\underline{\gamma}} \sum_{i=1}^{n}\left(l_{2 j i}\right)^{p /(p-1)}\right)^{p-1}\right] \\
&\left.\times \sum_{i=1}^{n} E\left|u_{i}(s)\right|^{p} d s+\left(\frac{\bar{J}_{j}}{\underline{b}_{j} \underline{\underline{\gamma}}}\right)^{p}\right\}
\end{aligned}
$$

Hence, by (22) and (23), we have that

$$
\begin{aligned}
& \sum_{i=1}^{n} E\left|u_{i}(t)\right|^{p}+\sum_{j=1}^{m} E\left|v_{j}(t)\right|^{p} \\
& \leq 6^{p-1} \sum_{i=1}^{n}\left\{\begin{array}{r}
\mathrm{e}^{-\underline{a}_{i}\left(t-t_{0}\right)} E\left|u_{i}\left(t_{0}\right)\right|^{p} \\
\\
\quad+\bar{a}_{i}^{p} \int_{t_{0}}^{t} \mathrm{e}^{-\underline{a}_{i}(t-s)} E\left|u_{i}(s)\right|^{p} d s
\end{array}\right.
\end{aligned}
$$

$$
\begin{gathered}
+\bar{a}_{i}^{p} \int_{t_{0}}^{t} \mathrm{e}^{-\underline{a}_{i}(t-s)} E\left|u_{i}\left(s-\tau_{i}(s)\right)\right|^{p} d s \\
+\int_{t_{0}}^{t} \mathrm{e}^{-\underline{a}_{i}(t-s)} \\
\times\left[\underline{a}_{i}^{1-p}\left(\frac{\bar{\gamma}}{\underline{\alpha}} \sum_{j=1}^{m}\left(\bar{c}_{i j} L_{j}^{f}\right)^{p /(p-1)}\right)^{p-1}\right. \\
+l_{p} n^{(p / 2)-1}\left(\frac{2 \underline{a}_{i}(p-1)}{p-2}\right)^{1-(p / 2)} \\
\left.\times\left(\frac{\bar{\gamma}}{\underline{\alpha}} \sum_{j=1}^{m}\left(l_{1 i j}\right)^{p /(p-1)}\right)^{p-1}\right] \\
\left.\times \sum_{j=1}^{m} E\left|v_{j}(s)\right|^{p} d s+\left(\frac{\bar{I}_{i}}{\underline{a}_{i} \underline{\alpha}}\right)^{p}\right\}
\end{gathered}
$$$$
+6^{p-1} \sum_{j=1}^{m}\left\{\mathrm{e}^{-\underline{b}_{j}\left(t-t_{0}\right)} E\left|v_{j}\left(t_{0}\right)\right|^{p}\right.
$$$$
+\bar{b}_{j}^{p} \int_{t_{0}}^{t} \mathrm{e}^{-\underline{b}_{j}(t-s)} E\left|v_{j}(s)\right|^{p} d s
$$$$
+\bar{b}_{j}^{p} \int_{t_{0}}^{t} \mathrm{e}^{-\underline{b}_{j}(t-s)} E\left|v_{j}\left(s-\sigma_{j}(s)\right)\right|^{p} d s
$$$$
+\int_{t_{0}}^{t} \mathrm{e}^{-\underline{b_{j}}(t-s)}
$$$$
\times\left[\underline{b}_{j}^{1-p}\left(\frac{\bar{\alpha}}{\underline{\gamma}_{i=1}^{n}} \sum^{n}\left(\bar{d}_{j i} L_{i}^{g}\right)^{p /(p-1)}\right)^{p-1}\right.
$$$$
+l_{p} m^{(p / 2)-1}\left(\frac{2 \underline{b}_{j}(p-1)}{p-2}\right)^{1-(p / 2)}
$$$$
\left.\times\left(\frac{\bar{\alpha}}{\bar{\gamma}} \sum_{i=1}^{n}\left(l_{2 j i}\right)^{p /(p-1)}\right)^{p-1}\right]
$$$$
\left.\times \sum_{i=1}^{n} E\left|u_{i}(s)\right|^{p} d s+\left(\frac{\bar{J}_{j}}{\underline{b}_{j} \underline{\gamma}}\right)^{p}\right\} .
$$

Set $V(t)=\sum_{i=1}^{n} E\left|u_{i}(t)\right|^{p}+\sum_{j=1}^{m} E\left|v_{j}(t)\right|^{p}$. By (24), we have that

$$
\begin{aligned}
V(t) \leq & 6^{p-1} \mathrm{e}^{-\theta\left(t-t_{0}\right)} V\left(t_{0}\right)+\int_{t_{0}}^{t} \epsilon \mathrm{e}^{-\theta(t-s)} V(s) d s \\
& +\int_{t_{0}}^{t} c \mathrm{e}^{-\theta(t-s)} V_{\delta}^{+}(s) d s+H,
\end{aligned}
$$


where $V_{\delta}^{+}(s)=\max _{-\delta \leq s \leq 0}|V(t+s)|, H=6^{p-1}$ $\max \left\{\left\{\max _{1 \leq i \leq n}\left(\bar{I}_{i} / \underline{a}_{i} \underline{\alpha}\right)^{p}\right\},\left\{\max _{1 \leq j \leq m}\left(\bar{J}_{j} / \underline{b}_{j} \gamma\right)^{p}\right\}\right\}$. By $\left(H_{4}\right)$ and Corollary 10 , the solutions of (10) are $p$-uniformly bounded and it also shows that the family of all solutions of (10) is $p$ point dissipative. Then, it follows from Lemma 5 that (10) has an $\omega$-periodic solution $\left(u_{1}^{*}(t), \ldots, u_{n}^{*}(t), v_{1}^{*}(t), \ldots, v_{m}^{*}(t)\right)^{T}$.

Suppose that $\left(u_{1}(t), \ldots, u_{n}(t), v_{1}(t), \ldots, v_{m}(t)\right)^{T}$ is an arbitrary solution of (10). Then it follows from (10) that

$$
\begin{aligned}
& d\left(u_{i}(t)\right.\left.-u_{i}^{*}(t)\right) \\
&=-a_{i}(t)\left(u_{i}\left(t-\tau_{i}(t)\right)-u_{i}^{*}\left(t-\tau_{i}(t)\right)\right) \\
&+ \prod_{t_{0}<t_{k}<t} \alpha_{i k}^{-1} \sum_{j=1}^{m} c_{i j}(t) \\
& \times {\left[f_{j}\left(\prod_{t_{0}<t_{k}<t} \gamma_{j k} v_{j}(t)\right)\right.} \\
&\left.-f_{j}\left(\prod_{t_{0}<t_{k}<t} \gamma_{j k} v_{j}^{*}(t)\right)\right] d t \\
&+ \prod_{t_{0}<t_{k}<t} \alpha_{i k}^{-1} \\
& \times {\left[\sum_{j=1}^{m} \sigma_{1 i j}\left(\prod_{t_{0}<t_{k}<t} \gamma_{j k} v_{j}(t)\right)\right.} \\
&\left.\quad-\sum_{j=1}^{m} \sigma_{1 i j}\left(\prod_{t_{0}<t_{k}<t} \gamma_{j k} v_{j}^{*}(t)\right)\right] d w_{1 j}(t), \\
& t \geq t_{0}, \quad i=1,2, \ldots, n,
\end{aligned}
$$

$$
\begin{aligned}
d\left(v_{j}(t)\right. & \left.-v_{j}^{*}(t)\right) \\
= & -b_{j}(t)\left(v_{j}\left(t-\sigma_{j}(t)\right)-v_{j}^{*}\left(t-\sigma_{j}(t)\right)\right) \\
+ & \prod_{t_{0}<t_{k}<t} \gamma_{j k}^{-1} \sum_{i=1}^{n} d_{j i}(t) \\
\times & {\left[g_{i}\left(\prod_{t_{0}<t_{k}<t} \alpha_{i k} u_{i}(t)\right)\right.} \\
& \left.-g_{i}\left(\prod_{t_{0}<t_{k}<t} \alpha_{i k} u_{i}^{*}(t)\right)\right] d t \\
+ & \prod_{t_{0}<t_{k}<t} \gamma_{j k}^{-1} \sum_{i=1}^{n}\left[\sigma_{2 j i}\left(\prod_{t_{0}<t_{k}<t} \alpha_{i k} u_{i}(t)\right)\right.
\end{aligned}
$$

$$
\begin{gathered}
\left.-\sigma_{2 j i}\left(\prod_{t_{0}<t_{k}<t} \alpha_{i k} u_{i}^{*}(t)\right)\right] d w_{2 i}(t), \\
t \geq t_{0}, \quad j=1,2, \ldots, m .
\end{gathered}
$$

Let $U(t)=\sum_{i=1}^{n}\left|u_{i}(t)-u_{i}^{*}(t)\right|^{p}+\sum_{j=1}^{m}\left|v_{j}(t)-v_{j}^{*}(t)\right|^{p}$. Proceeding as the proof of the existence of periodic solution of (10), from (26), we obtain that

$$
\begin{aligned}
U(t) \leq & 6^{p-1} \mathrm{e}^{-\theta\left(t-t_{0}\right)} U\left(t_{0}\right)+\int_{t_{0}}^{t} \epsilon \mathrm{e}^{-\theta(t-s)} U(s) d s \\
& +\int_{t_{0}}^{t} c \mathrm{e}^{-\theta(t-s)} U_{\delta}^{+}(s) d s .
\end{aligned}
$$

By $\left(H_{4}\right)$ and Corollary 10, the periodic solution of (10) is $p$-exponentially stable; that is, the periodic solution of (1) is $p$-exponentially stable. Therefore, (1) has an $\omega$-periodic solution, which is $p$-exponentially stable. This completes the proof of Theorem 12.

\section{An Example}

In this section, we present an example to illustrate the feasibility of our results obtained in the previous section.

Let $n=m=2$. Consider the following impulsive stochastic BAM neural network:

$$
\begin{aligned}
& d x_{i}(t)=\left[-a_{i}(t) x_{i}\left(t-\tau_{i}(t)\right)\right. \\
& \left.+\sum_{j=1}^{2} c_{i j}(t) f_{j}\left(y_{j}(t)\right)+I_{i}(t)\right] d t \\
& +\sum_{j=1}^{2} \sigma_{1 i j}\left(y_{j}(t)\right) d w_{1 j}(t), \\
& t \geq t_{0}, \quad t \neq t_{k}, \\
& x_{i}\left(t_{k}^{+}\right)=\alpha_{i k} x_{i}\left(t_{k}\right), \quad t>0, t=t_{k}, \\
& d y_{j}(t)=\left[-b_{j}(t) y_{j}\left(t-\sigma_{j}(t)\right)\right. \\
& \left.+\sum_{i=1}^{2} d_{j i}(t) g_{i}\left(x_{i}(t)\right)+J_{j}(t)\right] d t \\
& +\sum_{i=1}^{2} \sigma_{2 j i}\left(x_{i}(t)\right) d w_{2 i}(t), \\
& t \geq t_{0}, \quad t \neq t_{k}, \\
& y_{j}\left(t_{k}^{+}\right)=\gamma_{j k} y_{j}\left(t_{k}\right), \quad t>0, t=t_{k} \text {, }
\end{aligned}
$$


where $i, j=1,2$ and the coefficients are as follows:

$$
\begin{aligned}
& a_{1}(t)=1.15+0.05 \sin \frac{\pi}{2} t, \\
& a_{2}(t)=0.85+0.01 \cos \frac{\pi}{2} t, \\
& b_{1}(t)=0.86+0.01 \sin \frac{\pi}{2} t, \\
& b_{2}(t)=0.74+0.01 \cos \frac{\pi}{2} t \text {, } \\
& c_{11}(t)=0.04 \sin \frac{\pi}{2} t, \\
& c_{12}(t)=0.02 \cos \frac{\pi}{2} t, \\
& c_{21}(t)=0.03 \sin \frac{\pi}{2} t, \\
& c_{22}(t)=0.01 \sin \frac{\pi}{2} t, \\
& d_{11}(t)=0.04+0.03 \cos \frac{\pi}{2} t, \\
& d_{12}(t)=0.01 \sin \frac{\pi}{2} t, \\
& d_{21}(t)=0.05+0.01 \sin \frac{\pi}{2} t, \\
& d_{22}(t)=0.02 \cos \frac{\pi}{2} t, \\
& f_{1}(u)=0.03 \sin u, \\
& f_{2}(u)=0.04 \cos u, \\
& g_{1}(u)=0.05 \cos u, \\
& g_{2}(u)=0.07 \sin u, \\
& \sigma_{111}(u)=0.02 \sin u \text {, } \\
& \sigma_{112}(u)=0.03 \cos u \text {, } \\
& \sigma_{121}(u)=0.01 \sin u \text {, } \\
& \sigma_{122}(u)=0.04 \cos u \text {, } \\
& \sigma_{211}(u)=0.01 \cos u \text {, } \\
& \sigma_{212}(u)=0.04 \sin u \text {, } \\
& \sigma_{221}(u)=0.03 \sin u \text {, } \\
& \sigma_{222}(u)=0.04 \cos u \text {, } \\
& \tau_{1}(t)=\tau_{2}(t)=\sigma_{1}(t)=\sigma_{2}(t)=0.2+0.1 \sin \frac{\pi}{2} t, \\
& I_{1}(t)=I_{2}(t)=0.6 \sin \sqrt{2} t, \\
& J_{1}(t)=J_{2}(t)=0.5 \cos t, \quad i, j=1,2 \text {, } \\
& \alpha_{1 k}=\gamma_{1 k}=3^{\sin (k \pi / 2)} \text {, } \\
& \alpha_{2 k}=\gamma_{2 k}=3^{\cos (k \pi / 2)}, \quad k=1,2, \ldots
\end{aligned}
$$

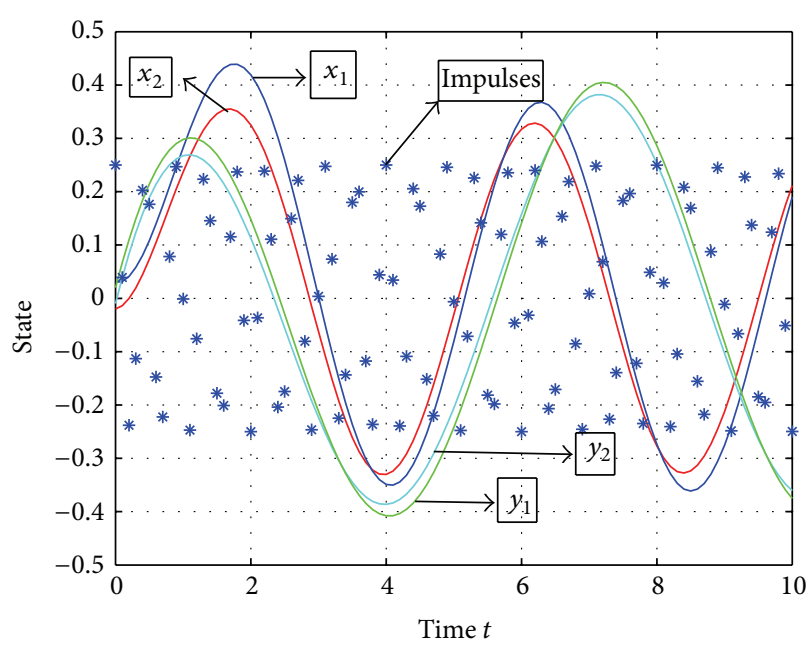

Figure 1: Transient states of stochastic BAM neural networks.

By calculating, we have

$$
\begin{aligned}
\bar{a}_{1}=1.2, & \underline{a}_{1}=1.1, \\
\bar{a}_{2}=0.86, & \underline{a}_{2}=0.84, \\
\bar{b}_{1}=0.87, & \underline{b}_{1}=0.85, \\
\bar{b}_{2}=0.75, & \underline{b}_{2}=0.73, \\
\bar{c}_{11}=0.04, & \bar{c}_{12}=0.02, \\
\bar{c}_{21}=0.03, & \bar{c}_{22}=0.01, \\
\bar{d}_{11}=0.07, & \bar{d}_{12}=0.01, \\
\bar{d}_{21}=0.06, & \bar{d}_{22}=0.02, \\
L_{1}^{f}=0.03, & L_{2}^{f}=0.04, \\
L_{1}^{g}=0.05, & L_{2}^{g}=0.07, \\
l_{111}=0.02, & l_{112}=0.03, \\
l_{121}=0.01, & l_{122}=0.04, \\
\bar{l}_{211}=0.01, & l_{212}=0.04, \\
l_{221}=0.03, & l_{222}=0.04, \\
\bar{\alpha}_{1}=3, & \underline{\alpha}=1 .
\end{aligned}
$$

Taking $p=3$, we can verify that all conditions of Theorem 12 are satisfied. Hence, (28) has a 4-periodic solution, which is 3-exponentially stable.

By the numerical simulation in Figures 1, 2, and 3 we can show that our results are plausible and effective. 


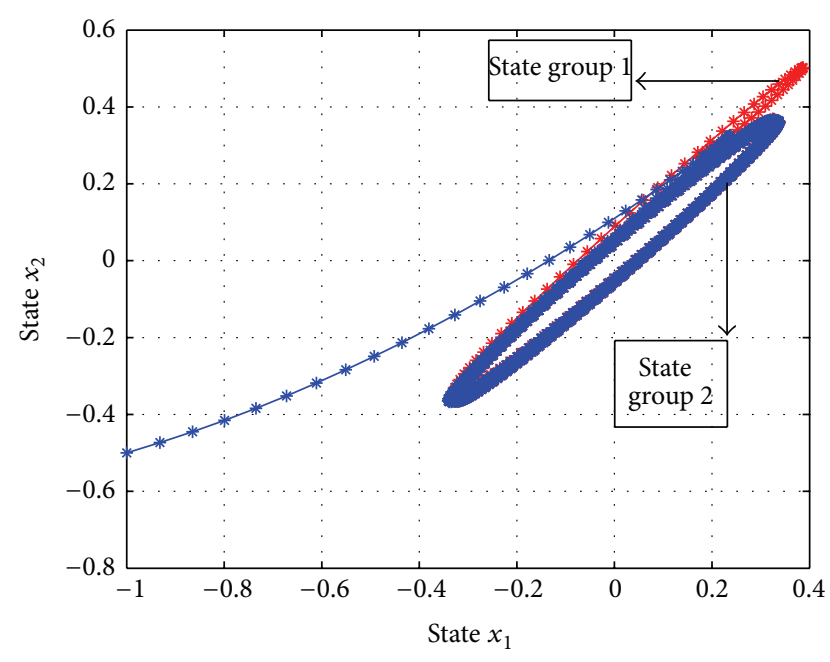

FIgURE 2: State response of $x_{1}$ and $x_{2}$.

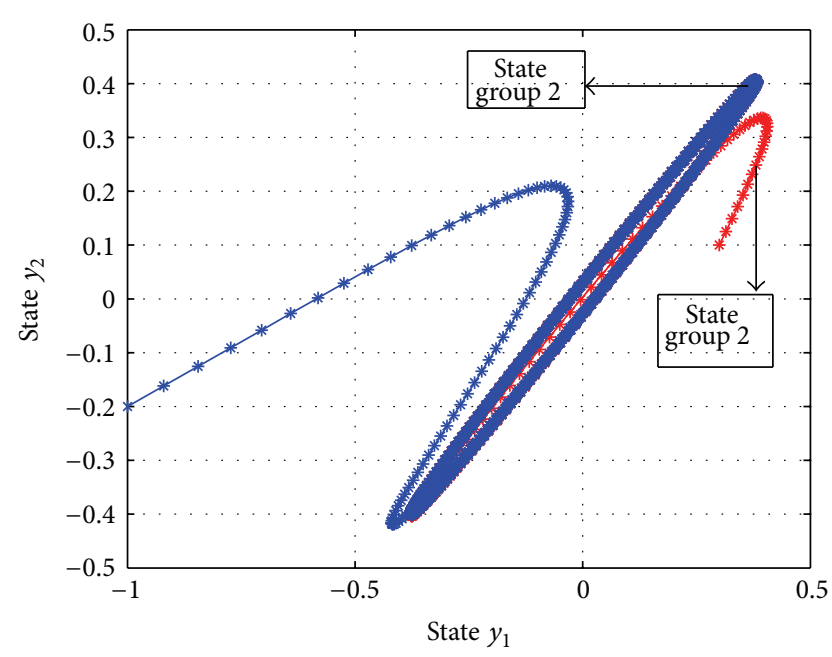

FIGURE 3: State response of $y_{1}$ and $y_{2}$.

\section{Acknowledgment}

This work is supported by the National Natural Science Foundation of People's Republic of China under Grant 10971183.

\section{References}

[1] B. Kosko, "Bidirectional associative memories," IEEE Transactions on Systems, Man and Cybernetics, vol. 18, no. 1, pp. 49-60, 1988.

[2] Y. K. Li, "Existence and stability of periodic solution for BAM neural networks with distributed delays," Applied Mathematics and Computation, vol. 159, no. 3, pp. 847-862, 2004.

[3] Q. Zhou, "Global exponential stability of BAM neural networks with distributed delays and impulses," Nonlinear Analysis: Real World Applications, vol. 10, no. 1, pp. 144-153, 2009.

[4] B. Zheng, Y. Zhang, and C. Zhang, "Global existence of periodic solutions on a simplified BAM neural network model with delays," Chaos, Solitons and Fractals, vol. 37, no. 5, pp. 1397-1408, 2008.
[5] Y. Xia, J. Cao, and M. Lin, "New results on the existence and uniqueness of almost periodic solution for BAM neural networks with continuously distributed delays," Chaos, Solitons and Fractals, vol. 31, no. 4, pp. 928-936, 2007.

[6] Y. K. Li, L. Yang, and W. Wu, "Periodic solutions for a class of fuzzy bam neural networks with distributed delays and variable coefficients," International Journal of Bifurcation and Chaos, vol. 20, no. 5, pp. 1551-1565, 2010.

[7] Y. K. Li and X. L. Fan, "Existence and globally exponential stability of almost periodic solution for Cohen-Grossberg BAM neural networks with variable coefficients," Applied Mathematical Modelling, vol. 33, no. 4, pp. 2114-2120, 2009.

[8] C. Liu, C. Li, and X. Liao, "Variable-time impulses in BAM neural networks with delays," Neurocomputing, vol. 74, no. 17, pp. 3286-3295, 2011.

[9] S. Haykin, Neural Networks, Prentice Hall, Upper Saddle River, NJ, USA, 1994.

[10] S. Blythe, X. Mao, and X. Liao, "Stability of stochastic delay neural networks," Journal of the Franklin Institute, vol. 338, no. 4, pp. 481-495, 2001.

[11] W. W. Su and Y. M. Chen, "Global robust stability criteria of stochastic Cohen-Grossberg neural networks with discrete and distributed time-varying delays," Communications in Nonlinear Science and Numerical Simulation, vol. 14, no. 2, pp. 520-528, 2009.

[12] H. Z. Yang and L. Sheng, "Robust stability of uncertain stochastic fuzzy cellular neural networks," Neurocomputing, vol. 73, no. 1-3, pp. 133-138, 2009.

[13] S. Zhu and Y. Shen, "Robustness analysis for connection weight matrices of global exponential stability of stochastic recurrent neural networks," Neural Networks, vol. 38, pp. 17-22, 2013.

[14] H. B. Bao and J. D. Cao, "Delay-distribution-dependent state estimation for discrete-time stochastic neural networks with random delay," Neural Networks, vol. 24, no. 1, pp. 19-28, 2011.

[15] Q. X. Zhu and J. D. Cao, "Exponential stability analysis of stochastic reaction-diffusion Cohen-Grossberg neural networks with mixed delays," Neurocomputing, vol. 74, no. 17, pp. 3084-3091, 2011.

[16] Y. Lv, W. Lv, and J. Sun, "Convergence dynamics of stochastic reaction-diffusion recurrent neural networks with continuously distributed delays," Nonlinear Analysis: Real World Applications, vol. 9, no. 4, pp. 1590-1606, 2008.

[17] B. Tojtovska and S. Janković, "On a general decay stability of stochastic Cohen-Grossbergneural networks with time-varying delays," Applied Mathematics and Computation, vol. 219, pp. 2289-2302, 2012.

[18] C. H. Wang, Y. G. Kao, and G. W. Yang, "Exponential stability of impulsive stochastic fuzzy reaction-diffusion Cohen-Grossberg neural networks with mixed delays," Neurocomputing, vol. 89, pp. 55-63, 2012.

[19] Q. X. Zhu and X. D. Li, "Exponential and almost sure exponential stability of stochastic fuzzy delayed Cohen-Grossberg neural networks," Fuzzy Sets and Systems, vol. 203, pp. 74-94, 2012.

[20] X. D. Li, "Existence and global exponential stability of periodic solution for delayed neural networks with impulsive and stochastic effects," Neurocomputing, vol. 73, no. 4-6, pp. 749758, 2010.

[21] G. Q. Peng and L. H. Huang, "Exponential stability of hybrid stochastic recurrent neural networks with time-varying delays," Nonlinear Analysis: Hybrid Systems, vol. 2, no. 4, pp. 1198-1204, 2008. 
[22] Y. Ou, H. Y. Liu, Y. L. Si, and Z. G. Feng, "Stability analysis of discrete-time stochastic neural networks with time-varying delays," Neurocomputing, vol. 73, no. 4-6, pp. 740-748, 2010.

[23] Q. K. Song and Z. D. Wang, "Stability analysis of impulsive stochastic Cohen-Grossberg neural networks with mixed time delays," Physica A, vol. 387, no. 13, pp. 3314-3326, 2008.

[24] K. Gopalsamy, Stability and Oscillations in Delay Differential Equations of Population Dynamics, Kluwer Academic, Dordrecht, The Netherlands, 1992.

[25] X. Li and J. Cao, "Delay-dependent stability of neural networks of neutral type with time delay in the leakage term," Nonlinearity, vol. 23, no. 7, pp. 1709-1726, 2010.

[26] X. Li, R. Rakkiyappan, and P. Balasubramaniam, "Existence and global stability analysis of equilibrium of fuzzy cellular neural networks with time delay in the leakage term under impulsive perturbations," Journal of the Franklin Institute, vol. 348, no. 2, pp. 135-155, 2011.

[27] X. Li, X. Fu, P. Balasubramaniam, and R. Rakkiyappan, "Existence, uniqueness and stability analysis of recurrent neural networks with time delay in the leakage term under impulsive perturbations," Nonlinear Analysis: Real World Applications, vol. 11, no. 5, pp. 4092-4108, 2010.

[28] P. Balasubramaniam, V. Vembarasan, and R. Rakkiyappan, "Leakage delays in T-S fuzzy cellular neural networks," Neural Processing Letters, vol. 33, no. 2, pp. 111-136, 2011.

[29] Q. T. Gan, R. Xu, and P. H. Yang, "Exponential synchronization of stochastic fuzzy cellular neural networks with time delay in the leakage term and reaction-diffusion," Communications in Nonlinear Science and Numerical Simulation, vol. 17, no. 4, pp. 1862-1870, 2012.

[30] S. Lakshmanan, J. H. Park, H. Y. Jung, and P. Balasubramaniam, "Design of state estimator for neural networks with leakage, discrete and distributed delays," Applied Mathematics and Computation, vol. 218, no. 22, pp. 11297-11310, 2012.

[31] B. W. Liu, "Global exponential stability for BAM neural networks with time-varying delays in the leakage terms," Nonlinear Analysis: Real World Applications, vol. 14, no. 1, pp. 559-566, 2013.

[32] Y. K. Li and Y. Q. Li, "Existence and exponential stability of almost periodic solution for neutral delay BAMneural networks with time-varying delays in leakage terms," Journal of the Franklin Institute, 2013.

[33] D. S. Li, X. H. Wang, and D. Y. Xu, "Existence and global pexponential stability of periodic solution for impulsive stochastic neural networks with delays," Nonlinear Analysis: Hybrid Systems, vol. 6, no. 3, pp. 847-858, 2012.

[34] R. Z. Has'minski1̌, Stochastic Stability of Differential Equations, Sijthoff and Noordhoff, Rockville, Md, USA, 1980.

[35] D. Xu, Y. Huang, and Z. Yang, "Existence theorems for periodic Markov process and stochastic functional differential equations," Discrete and Continuous Dynamical Systems, vol. 24, no. 3, pp. 1005-1023, 2009.

[36] F. Wu, S. Hu, and Y. Liu, "Positive solution and its asymptotic behaviour of stochastic functional Kolmogorov-type system," Journal of Mathematical Analysis and Applications, vol. 364, no. 1, pp. 104-118, 2010.

[37] A. Ichikawa, "Stability of semilinear stochastic evolution equations," Journal of Mathematical Analysis and Applications, vol. 90, no. 1, pp. 12-44, 1982. 


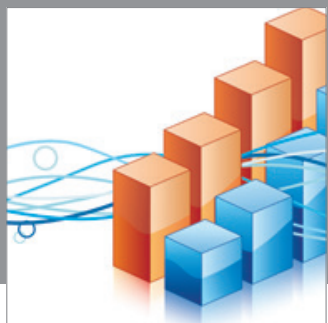

Advances in

Operations Research

mansans

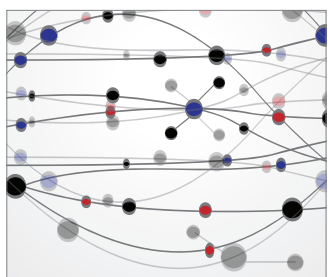

The Scientific World Journal
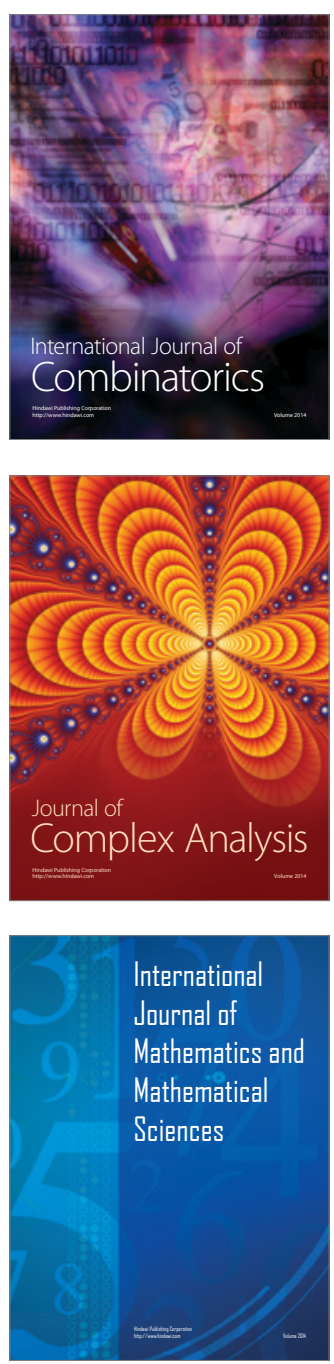
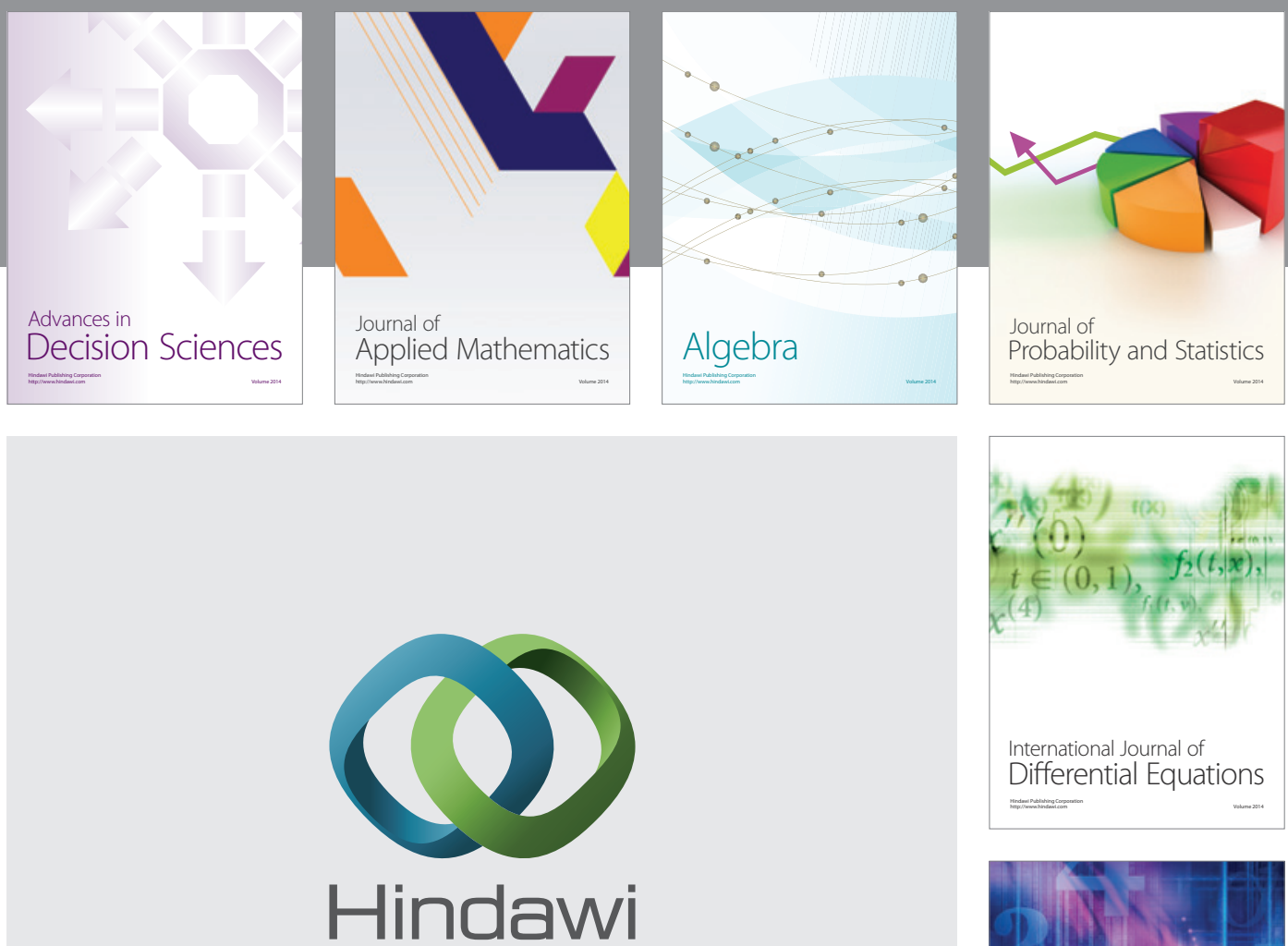

Submit your manuscripts at http://www.hindawi.com
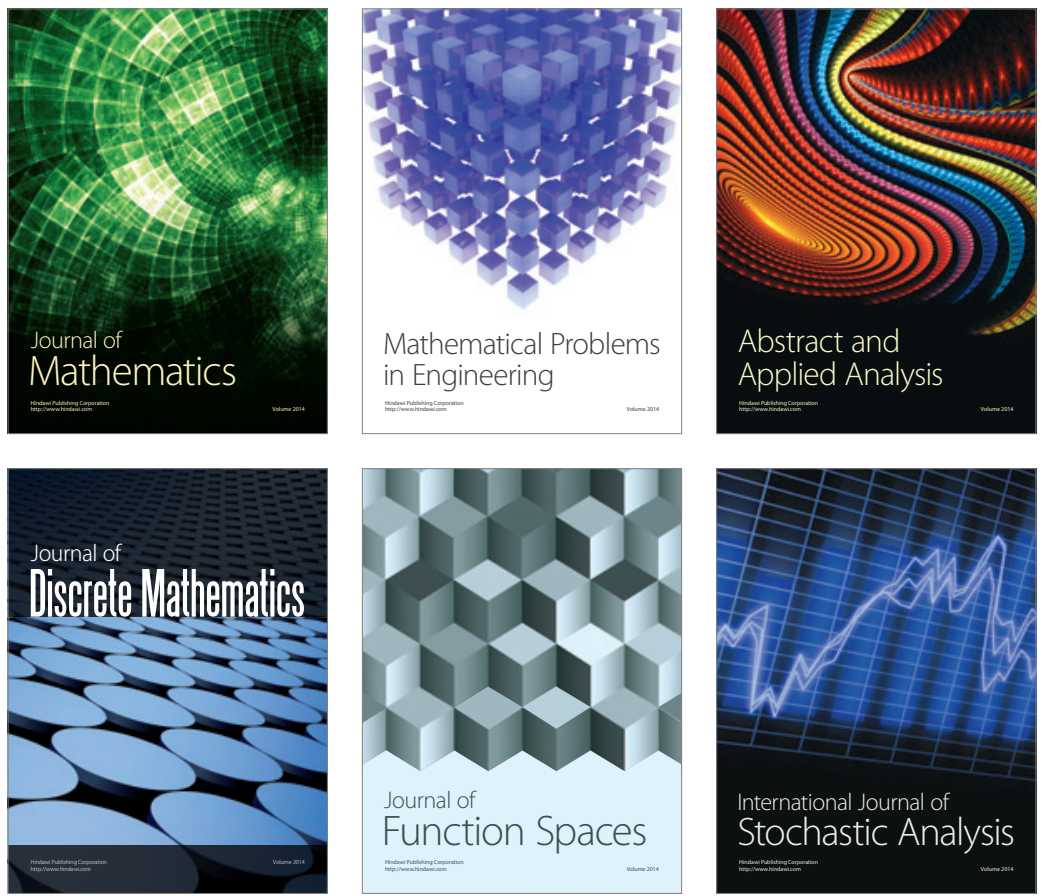

Journal of

Function Spaces

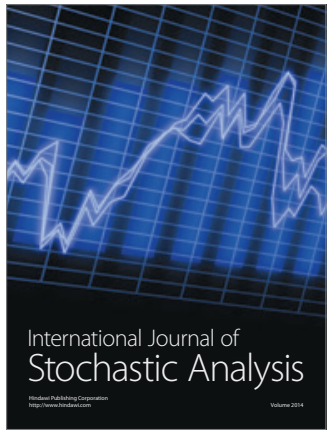

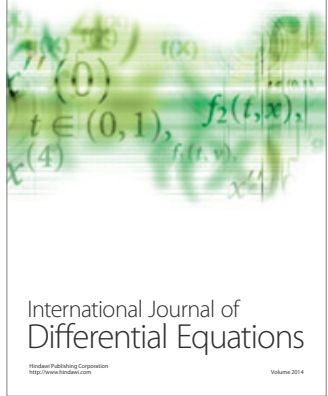
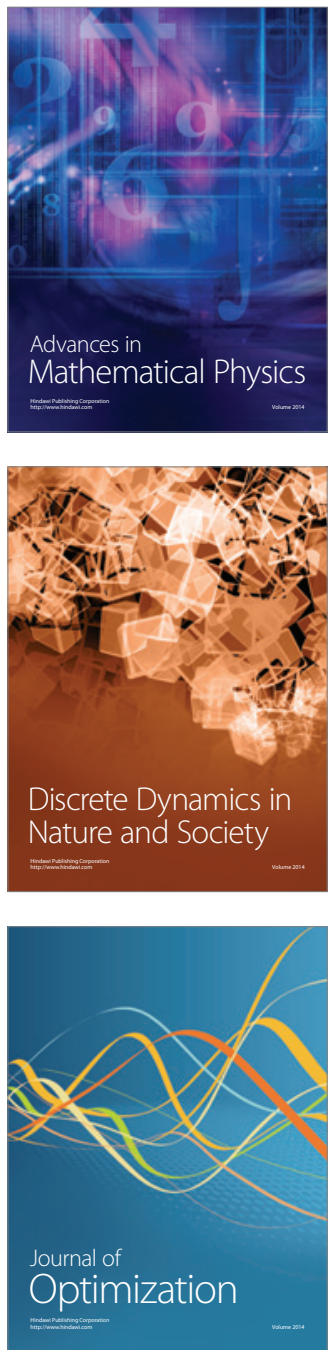